\title{
Synthesis Optimisation of Lysozyme Monolayer-Coated Silver Nanoparticles in Aqueous Solution
}

\author{
A. V. Yakovlev and O. Yu. Golubeva \\ Institute of Silicate Chemistry of Russian Academy of Sciences, Admirala Makarova Emb. 2, Saint Petersburg 199034, Russia \\ Correspondence should be addressed to A. V. Yakovlev; yakovlev.av@iscras.ru
}

Received 7 October 2014; Accepted 29 November 2014; Published 22 December 2014

Academic Editor: Subrata Kundu

Copyright (C) 2014 A. V. Yakovlev and O. Yu. Golubeva. This is an open access article distributed under the Creative Commons Attribution License, which permits unrestricted use, distribution, and reproduction in any medium, provided the original work is properly cited.

\begin{abstract}
This paper presents an optimisation of the synthesis of silver nanoparticles encapsulated in a biological shell. The synthesis was carried out in an aqueous solution of silver nitrate. Sodium borohydride was used as a reducing agent. Lysozyme served as a bioactive coating agent. The samples produced were studied using dynamic light scattering, transmission electron microscopy, and UV-Vis spectroscopy. The function of the dependence of the reagent ratio in obtained sols on optical properties is shown. Furthermore, the influence of the synthesis temperature, reactant ratio, and order of mixing on the particle size distribution parameters is shown. The optimal reagent mass ratio, $\mathrm{NaBH}_{4}: \mathrm{LYZ}: \mathrm{AgNO}_{3}=0.22: 0.77: 1$, is established. The resulting composition allows the synthesis of particles with a mean diameter of $18 \mathrm{~nm}$ and a bioshell thickness of $\approx 3.5 \mathrm{~nm}$. Moreover, the necessity of the synthesis optimisation and precise parameter control is clearly demonstrated.
\end{abstract}

\section{Introduction}

During the past few decades, many researchers [1] from various fields of science have been involved in the synthesis of noble metal nanoparticles for physics, chemistry, biology, and medicine applications [2]. This occurred because of the unique physicochemical properties of such materials, which make it possible to solve a wide range of problems in medicine, biology, catalysis, optics, and so forth [3-9].

The use of silver as an antibacterial agent is well known $[10,11]$. However, ionic silver exhibits high toxicity properties to human cells [11] and, at present, its application in the ionic form is extremely restricted. One of the ways to solve the usability issue with silver is to utilise silver in nanoparticles, rather than in the ionic form. This method not only avoids the toxicity concerns but also yields unique material properties, such as providing a selective action on microorganisms through bioactive agents by targeted delivery on the surface of carrier nanoparticles [12]. Chemical and photochemical reductions in solutions are the simplest and most common methods of silver nanoparticle synthesis. These methods allow for the generation of small nanoparticles with a definite shape [13]. Furthermore, the use of composites based nanoparticle is common and has a good potential in everyday use [14]. However, the issue of the instability of the obtained sols has not been completely solved. Moreover, for medical applications, the commonly used stabilisers may be very toxic or allergenic for humans. Furthermore, strongly diluted solutions are used in conventional synthesis techniques for the production of particles smaller than $50 \mathrm{~nm}$. This results in a low quantity of nanoparticles [15] in solutions, which makes the study of such objects' properties and their practical application difficult. Therefore, in this paper, a more promising particle stabilisation technique, which is directly related to particle synthesis optimisation and functionalisation by creating bioactive protective shells, will be described. In this case, the obtained particles have a metal core nanoparticle and a biomolecular shell. The synthesis of these substances (bioconjugates) removes some of the problems that arise in the synthesis of nanoparticles in solutions, for example, the issues of stability and nanoparticle dispersion. As demonstrated by Eby et al. [16], such ferments as lysozyme can not 
only build up strong shells on the nanoparticle surface but also serve as a catalyst in the photochemical synthesis of silver nanoparticles. Despite the simplicity of this technique, it has certain shortcomings and has clear disadvantages for industrial implementation [7]. The main difficulty stems from using noxious and/or organic media for the production of nanoparticles. The purification of such sols is a very timeand-energy-consuming process.

The most critical parameter for the use of bioconjugates is their size [17-19]. The presence of even a few particles larger than $60 \mathrm{~nm}$ can make it impossible to predict the biological effect of bioconjugates or to compare the results of their study. Commonly, to reduce the negative effects of particle size differences and to achieve a nearly monomodal size distribution, various complex purification methods are used $[20,21]$. As mentioned previously, these methods are laborious and greatly reduce the amount of nanoparticles in sols. Some investigators use different techniques to control particle size, such as micellar synthesis or stabilisation in matrices [22-24].

The proposed synthesis method is based on the formation of protein lysozyme monolayer-coated silver nanoparticles in an aqueous solution of a three-component synthesis system of source substances $\left(\mathrm{LYZ}: \mathrm{NaBH}_{4}: \mathrm{AgNO}_{3}\right)$.

Our main objective is the study of the basic synthesis regularities and the selection of the optimal synthesis conditions, which has not been reported previously [25-27]. The selected conditions should produce silver nanoparticles smaller than $50 \mathrm{~nm}$ in size, with a narrow particle size distribution.

Synthesis using the suggested conditions involves certain shortcomings and problems to be solved. As described previously, silver nitrate interacts with lysozyme in sunlight. This interaction results in the production of nanoparticles coated with a lysozyme shell. The size of the particles produced in the aqueous solution is $60-80 \mathrm{~nm}$, and the particle size distribution may differ considerably from sample to sample. For the proposed method, this side effect is a negative contribution to the particle size distribution that should be avoided or accounted for in the experiments. Because the reaction proceeds in an aqueous medium, it is impossible to completely exclude the solvent effect [28]. The experiment is also complicated by the concurrent sodium borohydride hydrolysis reaction. In practice, excess sodium borohydride is used to reduce the negative effect of hydrolysis. For many years, this procedure has been well-proven. However, this method shows a significant disadvantage: the use of excess sodium borohydride causes an increase in the concentration of its hydrolysis products $\left(\mathrm{NaBO}_{2}\right)$, which may strongly affect the long-term stability of the system. Therefore, it is necessary to carry out a study of the optimal weight ratio of the initial reagents that allows stable hydrosols production with the required physicochemical parameters while maintaining long-term stability. Despite the disadvantages mentioned above, we assume that the considered synthesis system is convenient for a study of the nanoparticle biocoating phenomena in question and can be adapted for practical use in medicine.

We attempt to demonstrate the importance of an in-depth investigation of the processes and conditions of nanoparticle formation and substantiate the effect of precise synthesis performance on the basic physicochemical parameters of the lysozyme-coated nanoparticles. The data obtained will allow the acquired knowledge to be extended to other systems and will provide researchers with a more convenient, practical, and cost-effective method of nanoparticle synthesis.

\section{Materials and Methods}

2.1. Reagents. All the reagents, hen egg lysozyme (LYZ, $\approx 70000$ units/mg, Sigma-Aldrich, USA), sodium borohydride $\left(\mathrm{NaBH}_{4}, 99 \%\right.$, Sigma-Aldrich, USA), and silver nitrate $\left(\mathrm{AgNO}_{3}, 99.9 \%\right.$, Khimmedsintez, Russia), were of a high purity grade and were used without additional purification. To prepare the solutions, we used deionised water produced from distilled water using a Vodolei setup (NPP Khimelektronika, Russia) with specific conductivity no higher than $0.2 \mu \mathrm{S} / \mathrm{cm}$. The experiments were performed in sterile, disposable $2 \mathrm{~mL}$ microvials.

2.2. Methods of Analysis and Instrumentation. To prepare the stock solutions, volumetric flasks of first-class accuracy, pretreated with a chromic mixture and washed no less than ten times with deionised water, were used. The temperature was controlled in a CH-100 thermostat (Biosan, Latvia) with holes for the microvials and a built-in Peltier element that set the temperature range at $(-20-100) \pm 0.1^{\circ} \mathrm{C}$. The reagents were mixed on a V-1 vortex (Biosan, Latvia). Particle sizes were measured with the dynamic light scattering method using a Nanotrac Ultra instrument (Microtrac, USA) with no special sample preparation. The particle size distribution was computed using the Microtrac Flex 10.6.1 software (Microtrac, USA). Additionally, transmission electron microscopy (TEM) was used to measure the particle sizes on a Jem 2100F microscope (JEOL, USA) at $\mathrm{U}_{\mathrm{ac}}$ up to $200 \mathrm{kV}$. The samples were prepared using a standard carbon replica technique.

Micrographs were processed using the Gimp 2.8 graphics editor. The particle size distribution graph was constructed and approximated by the Gauss method using the Python 2.7.5 programming language and the SciPy library SciPy [29]. A spectroscopic study was carried out on a UV-Vis UV-2600 spectrophotometer (Shimadzu, Germany) using cells with an optical path length of $5 \mathrm{~mm}$. Before measurements, the samples were diluted 10 -fold, and the total absorption spectra were measured. For all the samples, the position of the absorbance peak and its area were determined.

The postsynthesis content of the residual ionic silver was measured with ion-selective analysis using an Ekspert-001 instrument (Ekoniks-Ekspert, Russia).

2.3. Nanoparticle Synthesis. The nanoparticles were synthesised in aqueous solutions using a chemical reduction of silver nitrate by sodium borohydride in the presence of lysozyme.

Solutions with the following concentrations were prepared for experiments: $C_{\mathrm{AgNO}_{3}}=10 \mathrm{mM}, C_{\mathrm{NaBH}_{4}}=20 \mathrm{mM}$, and $C_{\mathrm{LYZ}}=3 \mathrm{mg} / \mathrm{mL}$. A fresh sodium borohydride solution was prepared for each series of experiments, and it was immediately cooled to a temperature of $0^{\circ} \mathrm{C}$. The necessary 
amounts of the sodium borohydride and silver nitrate solutions were transferred to the microvials by an autopipette. Then, the lysozyme solution was added (half of the required amount to the silver nitrate solution and the other half to the sodium borohydride solution) to obtain the desired $\mathrm{NaBH}_{4}$ : LYZ: $\mathrm{AgNO}_{3}$ weight ratio. The lysozyme and silver nitrate solution mixture was shielded from sunlight. The reagent mixing weight ratio was varied in such a way that the amount of reduced silver and the final volume of the solution remained constant. This was achieved by adding the required volume of deionised water.

Before mixing, the solutions were cooled or heated to the required temperature in the thermostat for ten minutes to complete temperature stabilisation. Then, the solutions were mixed and, after synthesis, naturally equilibrated to room temperature. Obtained samples were analysed using the previously described methods.

\section{Results and Discussion}

It was shown that the results' reproducibility, sol stability, and single-mode particle size distribution could only be obtained with the proper order of reagent mixing as described above. All other methods of solution preparation resulted in a bimodal particle size distribution or a very broad particle size distribution. Moreover, the solution mixture stirring rate did not play any part within the tested range of 700-3500 rpm.

Let us consider the effect of the $\mathrm{NaBH}_{4}: \mathrm{AgNO}_{3}$ weight ratio on the sol parameters. According to the experimental data, an excess of sodium borohydride resulted in instantaneous particle precipitation. Therefore, our study was conducted within the weight ratio $\mathrm{NaBH}_{4}: \mathrm{AgNO}_{3}<1 \mathrm{~g} / \mathrm{g}$. An excess of silver nitrate up to the weight ratio $\mathrm{NaBH}_{4}: \mathrm{AgNO}_{3}$ $=0.1 \mathrm{~g} / \mathrm{g}$ did not significantly affect the particles size or their distribution. However, when exposed to light, the excess silver nitrate reduced to form particles 80 to $100 \mathrm{~nm}$ in size, which affected the general particle size distribution. Therefore, for the next study, we chose the ratio $\mathrm{NaBH}_{4}: \mathrm{AgNO}_{3}=$ $1 \mathrm{~g} / \mathrm{g}$. At this precursor-to-reducing-agent ratio, the residual amount of silver ions does not exceed a concentration of $10^{-6} \mathrm{~mol} \cdot \mathrm{L}^{-1}$ in all the sample series.

Let us consider the effect of the LYZ: $\mathrm{AgNO}_{3}$ ratio depicted in Figure 1. The experiments were carried out at the equivalent $\mathrm{NaBH}_{4}: \mathrm{AgNO}_{3}$ ratio and a solution temperature of $0^{\circ} \mathrm{C}$. The obtained dependence can be broken up into the following three main weight ratio ranges.

(1) The $0.0-0.6 \mathrm{~g} / \mathrm{g}$ range corresponding to lysozyme deficiency. The nanoparticles are stabilised at the initial growth stage. The uncoated particles undergo uncontrolled growth and aggregation phases.

(2) The $0.6-1.0 \mathrm{~g} / \mathrm{g}$ range corresponding to the stability condition with the optimum reagent ratio. Within this range, a complete lysozyme monolayer coating of the particles occurs.

(3) The range $>1.0 \mathrm{~g} / \mathrm{g}$ corresponding to particle conjugation completion and the onset of secondary lysozyme layer formation over the monolayer. This process

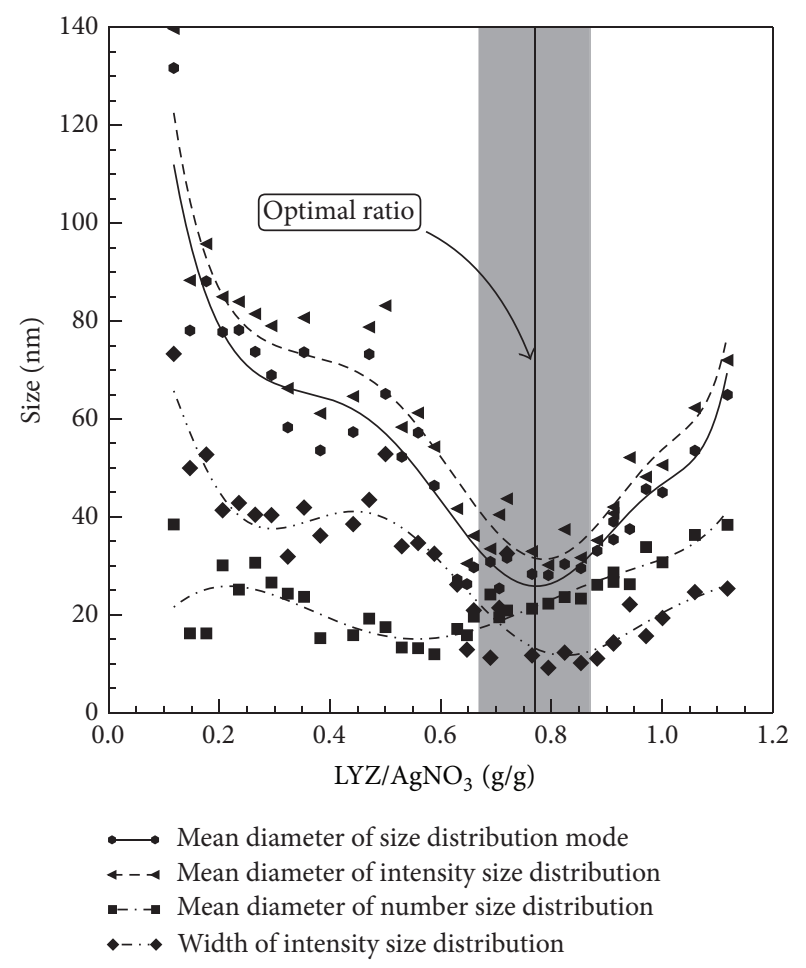

FIGURE 1: The effect of lysozyme-silver nitrate weight ratio on the parameters of the particle size distribution, obtained by dynamic light scattering.

results in an increase in the hydrodynamic radius, which is revealed by the dynamic light scattering method and by a change in the optical properties of the particles.

To better understand and interpret the data, let us consider each dependency individually. As follows from Figure 1, the curves representing the mean particle diameters corresponding to the major mode of the particle size distribution determined by the measured light scattering intensity and to the total particle size distribution virtually coincide and exhibit similar behaviour, which unambiguously excludes the bimodal nature of the particle size distribution. As mentioned earlier, the particle size is of vital importance, even if the number of such particles is insignificant.

It should be noted that the width of the intensity size distribution is similar to that of the curves discussed above, and their minimum lies at the ratio $\mathrm{LYZ}: \mathrm{AgNO}_{3} \approx 0.77 \mathrm{~g} / \mathrm{g}$. The total distribution width at this region is less than $20 \mathrm{~nm}$. Based on the importance of this parameter, it would be erroneous to consider only the mean particle diameter of the size distribution by number. In this case, the application of the dynamic light scattering technique has a great advantage because the larger particles can scatter light much better and contribute more to the distribution by intensity than smaller particles. Through the examination of the number distribution of the particle sizes shown in Figure 1, it can be observed that the values for all the ratios are similar, with a slight bend at the weight ratio of $0.6 \mathrm{~g} / \mathrm{g}$ and an increase after 
a weight ratio of $0.9 \mathrm{~g} / \mathrm{g}$. This distribution is closest to the actual distribution, which can be obtained by transmission electron microscopy without taking into account the difference between the hydrodynamic diameter of the particles with an organic shell and the actual diameter of the corenanoparticles observed by transmission electron microscopy images. From the data obtained, the best stabilisation and optimal consumption of reagents in conjunction with the completely homogeneous properties of the obtained products occur in a narrow interval of reactant ratios, with a weight ratio of approximately $\mathrm{NaBH}_{4}: \mathrm{LYZ}: \mathrm{AgNO}_{3}=0.22: 0.77: 1$. The resulting value is in good agreement with data obtained by Eby and others but has greater accuracy [16].

In addition to dynamic light scattering, UV-Vis spectroscopy provides very important information about the optical properties of the obtained bioconjugates. The spectrum presented in Figure 2 clearly exhibits the absorption band with a peak at a wavelength of $404 \mathrm{~nm}$.

It is known that, for such small particles, the absorption wavelength should be in the range of 398-400 nm [30]. For the bioconjugates, the absorption wavelength shift may be evidence of biomolecular interaction with the surface of a nanoparticle.

The absorption spectrum may be described based on the following three main characteristics:

(i) the position of the peak associated with the particle size,

(ii) the absorbance intensity directly related to the number of nanoparticles,

(iii) the peak area that can implicitly indicate the presence of larger nanoparticles.

In Figure 3, these parameters are presented as a function of the reagent ratio.

It is worth mentioning that the visual colour of the solution on the orientation, in the case of systems having a sufficiently high concentration of nanoparticles, has no practical utility and can be wrongly interpreted. During synthesis, the samples immediately became dark yellow in colour and nearly opaque after adding the reducing agent. Therefore, determination of the "good" samples from "bad" in this manner is not possible. However, when the sol is diluted, a characteristic yellow colour appears.

If we consider that the amount of reduced silver ions in the solutions was kept constant and equal to the molar concentration of sodium borohydride solution, the intensity of absorption is fully responsible for the amount of nanoparticles that exhibit plasmon resonance.

The curves shown in Figure 3 describe the change of the peak area, the intensity, and the wavelength of the peak maximum. Maximum intensity undergoes changes stronger than all the other parameters. The peak of this curve is located in the area-to-mass ratio of lysozyme silver nitrate $\approx 0.77 \mathrm{~g} / \mathrm{g}$, which corresponds to the optimal weight ratio obtained by dynamic light scattering. This finding indicates that the most efficient light absorbing sols are in the region where the optimal weight ratio is.

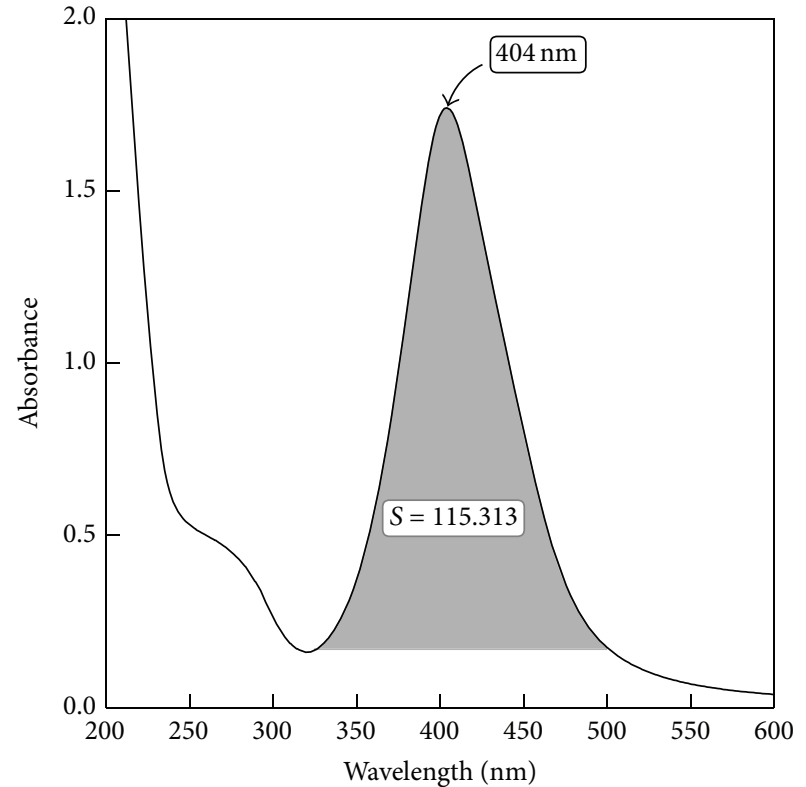

FIGURE 2: UV-Vis absorption spectrum of the bioconjugate sol with optimal parameters of synthesis: $\mathrm{NaBH}_{4}: \mathrm{LYZ}: \mathrm{AgNO}_{3}=$ $0.22: 0.77: 1$ and $t=0^{\circ} \mathrm{C}$. $S$-area under the curve.

The curve of the peak wavelengths has a more intricate shape and cannot be easily explained. Referring again to Figure 1, we can conclude that with weight ratios less than $0.4 \mathrm{~g} / \mathrm{g}$ there is a slow decrease in particles size and they begin to absorb light. Additionally, the particle size is large due to the incomplete shell, and the wavelength of maximum absorption is shifted to the red region of the spectrum (which may be caused by influence of uncoated particles). In this region of weight ratios, the results may fluctuate strongly in the experiments because uncontrolled particle aggregation occurs, and the particle size distribution is far from optimum. When the weight ratio is increased beyond $0.4 \mathrm{~g} / \mathrm{g}$, the influence of conjugated nanoparticles is noticeable.

At weight ratios of approximately $0.77 \mathrm{~g} / \mathrm{g}$, complete coverage of the particles occurs. The wavelength of the peak shifts slightly due to the interaction of lysozyme with the surface of the particle. Increasing the amount of lysozyme further leads to a multilayer coating and a change in the optical properties of the particles. However, it should be noted that the variation in the wavelength corresponding to the absorbance peak position is very small and occurs within 399-407 $\mathrm{nm}$.

The curve of the peak area in Figure 3 definitively confirms previous findings. At weight ratios less than $0.4 \mathrm{~g} / \mathrm{g}$, the peak area is formed due to the peak broadening caused by the presence of large-sized particles. At a weight ratio of $\approx 0.5 \mathrm{~g} / \mathrm{g}$, the peak narrows and then its area only increases due to the increasing absorbance intensity (zone of intensity growth in Figure 3).

To explain the obtained result more easily, let us examine Figure 4. It is clear that, due to the lack of lysozyme in the system, the absorption spectrum has a broad base and relatively weak intensity. The broadening is also noticeable in 


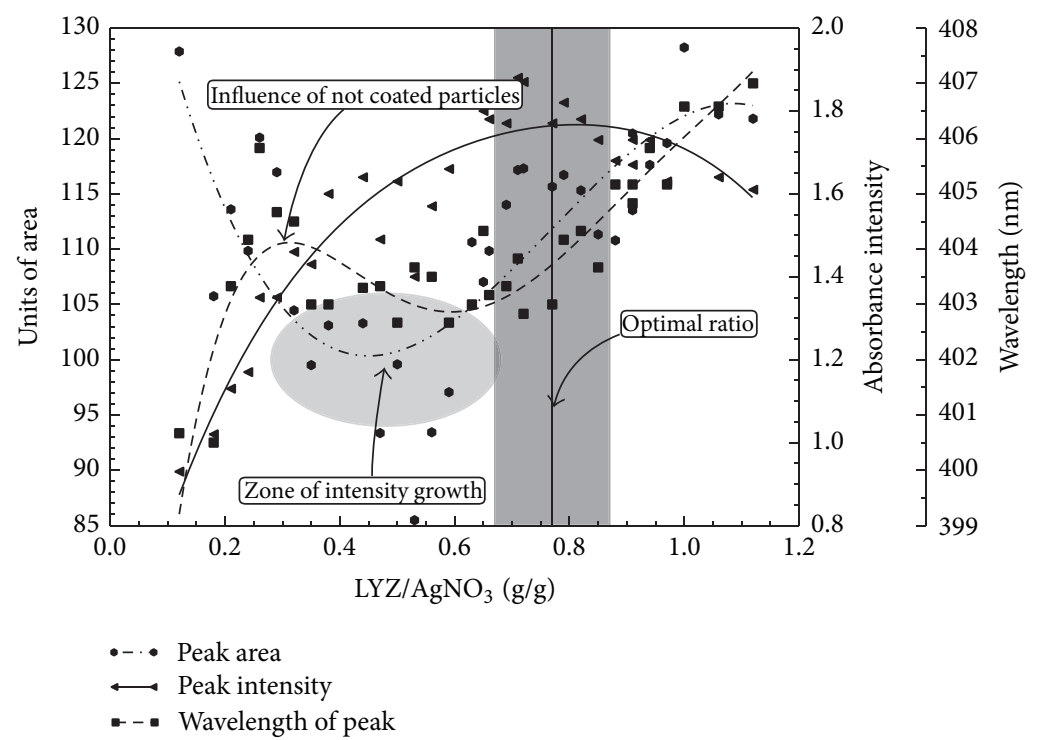

FIGURE 3: Absorbance peak area, its intensity, and the wavelength corresponding to the peak position as functions of the $\mathrm{NaBH}_{4}: \mathrm{LYZ}_{\mathrm{Z}}: \mathrm{AgNO}_{3}$ $=0.22: 0.77: 1$ and $t=0^{\circ} \mathrm{C}$.

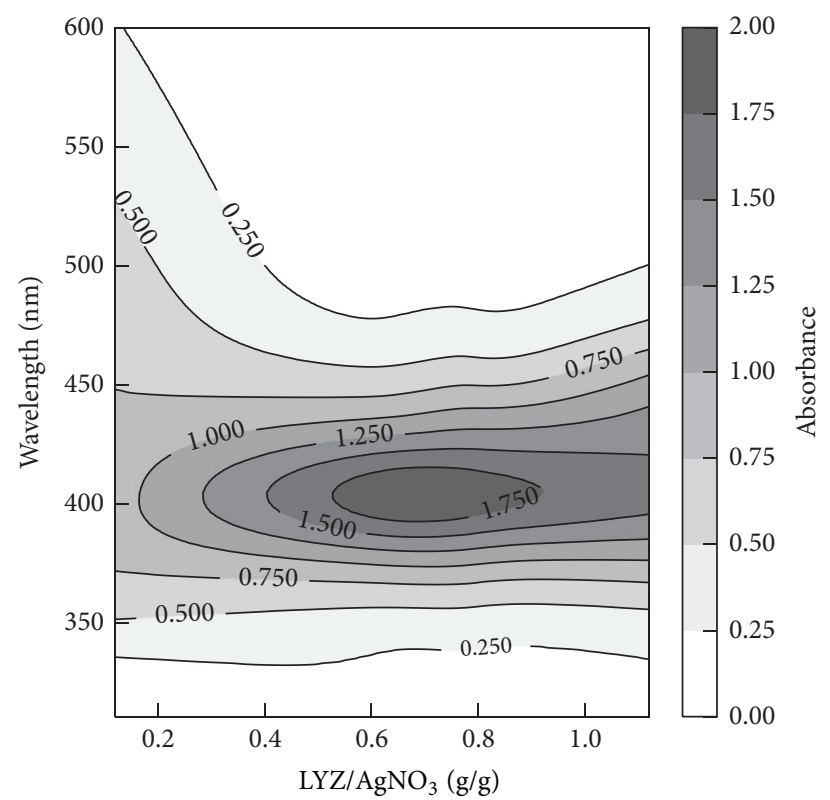

FIGURE 4: Contour plot displaying changes in the nature of the UVVis transmission spectra curve as a function of the $\mathrm{LYZ}: \mathrm{AgNO}_{3}$ weight ratio and at $t=0^{\circ} \mathrm{C}$.

the range of the high $\mathrm{LYZ}: \mathrm{AgNO}_{3}$ weight ratio. The peak of this curve, situated in the $0.6-0.8 \mathrm{~g} / \mathrm{g}$ weight ratio range, is where the peak base is the narrowest and has its maximum intensity.

Figure 5 shows a micrograph of a sample prepared at the optimum reagent ratio. This micrograph shows that the particles are spaced apart, and no large aggregations are observed. A particle with a regular spherical shape is observed in the higher-resolution micrograph in the inset in Figure 5. However, it appears impossible to determine

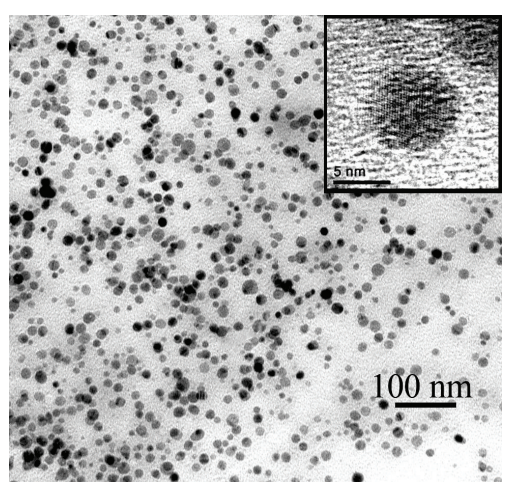

FIGURE 5: Micrograph of a sample synthesised in optimal parameters of synthesis at the weight ratio $\mathrm{NaBH}_{4}: \mathrm{LYZ}: \mathrm{AgNO}_{3}=0.22: 0.77: 1$, $t=0^{\circ} \mathrm{C}$.

the parameters of the biological shell from the micrographs because of its small size and poor contrast. But on the micrograph (Figure 6) of sol, obtained in lack of lysozymes which have larger size of nanoparticles, the shell is easier to capture and measure. From the data obtained, the size of the shell is $\approx 3.7 \mathrm{~nm}$.

The comparison of particle size distributions in Figure 7(a), obtained by different methods (DLS and TEM), shows that certain curves shift relative to each other.

Shift values between the curves at Figure $7(b)$ reflect the difference between the hydrodynamic diameter of the bioconjugate $(d=25.1 \mathrm{~nm}$ and $\mathrm{SD}=11.2 \mathrm{~nm})$ and the size of the metallic core-nanoparticles $(d=17.0 \mathrm{~nm}$ and $\mathrm{SD}=7.3 \mathrm{~nm}$ ); this difference is $\approx 7 \mathrm{~nm}$. Consequently, the thickness of the coating on the particle surface is $\approx 3.5 \mathrm{~nm}$, considering that the error of the methods matches the lysozyme hydrodynamic size, which should be $\approx 2-5 \mathrm{~nm}$ [28], depending on the properties of the solution, the location 


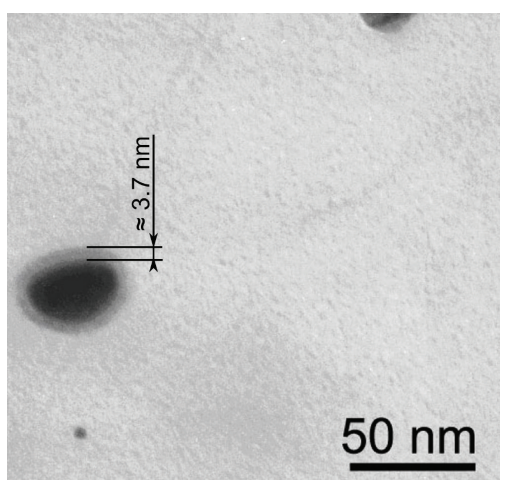

FIGURE 6: Micrograph of a sample synthesised in nonoptimal weight ratio $\mathrm{NaBH}_{4}: \mathrm{LYZ}: \mathrm{AgNO}_{3}=0.22: 0.6: 1, t=0^{\circ} \mathrm{C}$.

of a molecule on the surface of its structure, and other physicochemical properties. Furthermore, conformational changes in the lysozyme structure [31-34] and interaction with the solution occur on the surface, which may strongly affect the measured shell thickness. Furthermore, this value is in excellent agreement with previous results obtained by transmission electron microscopy (Figure 6). Special attention should be given to the width of the distribution curves under consideration. Dynamic light scattering curves have a long tail of up to $\approx 50 \mathrm{~nm}$, which is absent in the data from the transmission electron microscopy. This discrepancy may be due to the fixing of the total hydrodynamic radius of small groups of the coated particles. However, another explanation is that a large sample of particles with dynamic light scattering and the registration of small amount of larger particles is taking place.

During the experiments, it was found that, under optimal conditions, the stirring speed does not affect the size of the obtained particles. However, the order of mixing is of great importance. Narrow particle size distribution and a small particle size is obtained only by the method of 8 , which was described earlier.

Figure 8 shows the impact of the synthesis temperature on particle size distribution characteristics. From this it follows that the temperature of synthesis does not influence the numerical distribution of the particle sizes. However, it does have a very strong influence on the particle size distribution width and the intensity distribution. As the synthesis temperature rises, a second mode appears in the distribution corresponding to the particle sizes within the $80-100 \mathrm{~nm}$ range. This finding corresponds to the quantity of increase of large particles, but they still have an extremely small quantity compared to the total. This is directly associated with the effect of the sodium borohydride hydrolysis reaction and does not take into account changes in the $\mathrm{NaBH}_{4}: \mathrm{AgNO}_{3}$ weight ratio. Consequently, the synthesis temperature is an extremely important condition that must be monitored to ensure that no impurities of large particles occur in the sols. It is worth mentioning that, after the stabilisation process, the resulting sol nanoparticles have a wide temperature range of stability. In these experiments, the particle size and optical properties are not changed in the temperature range from 0

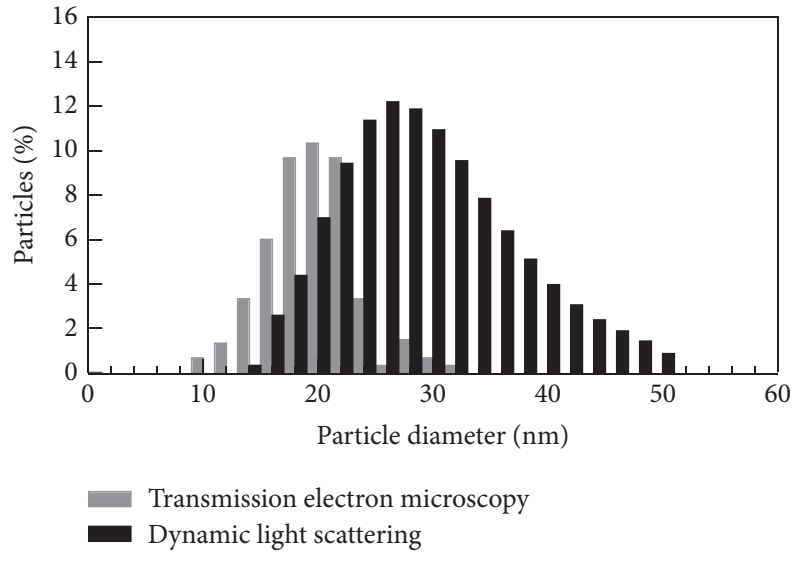

(a)

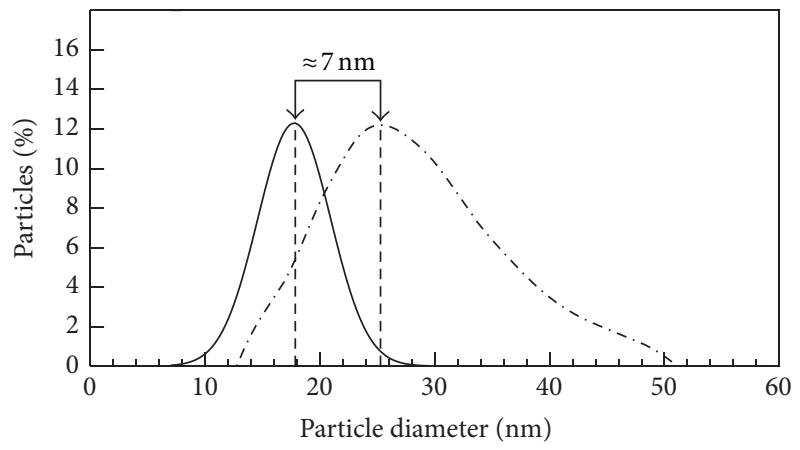

Gaussian approximation
_..- Transmission electron microscopy
-. Dynamic light scattering

(b)

FIgURE 7: Comparison of the particle size distributions obtained by the dynamic light scattering and TEM methods (analysis of more than 800 particles). (a) Actual distributions; (b) Gaussian approximation with the distribution mode indicated.

to $60^{\circ} \mathrm{C}$. The only limitations of the temperature application to the sol can be that a decrease in activity over long periods ( $>60 \mathrm{~min}$ ) impacts temperatures $>60^{\circ} \mathrm{C}$, at which the enzyme inactivation occurs [35]. Presumably, with partial destruction of the shell, there is a reduction of the stabilising ability. However, to our knowledge, up to a temperature of $90^{\circ} \mathrm{C}$ and an exposure time $<60 \mathrm{~min}$, changes in the parameters of the sol were not observed.

\section{Conclusion}

Our investigation has demonstrated the high importance of a comprehensive study of nanoparticle synthesis conditions.

The data obtained on $\mathrm{NaBH}_{4}: \mathrm{LYZ}: \mathrm{AgNO}_{3}$ aqueous solution systems show that the proper selection of the reagent ratio, synthesis temperature, and mixing speed makes it possible to obtain outstanding results on such qualitative parameters as the sol stability, mean particle size, and the size distribution width. In this study, we succeeded in establishing basic synthesis regularities that provide production of hybrid 


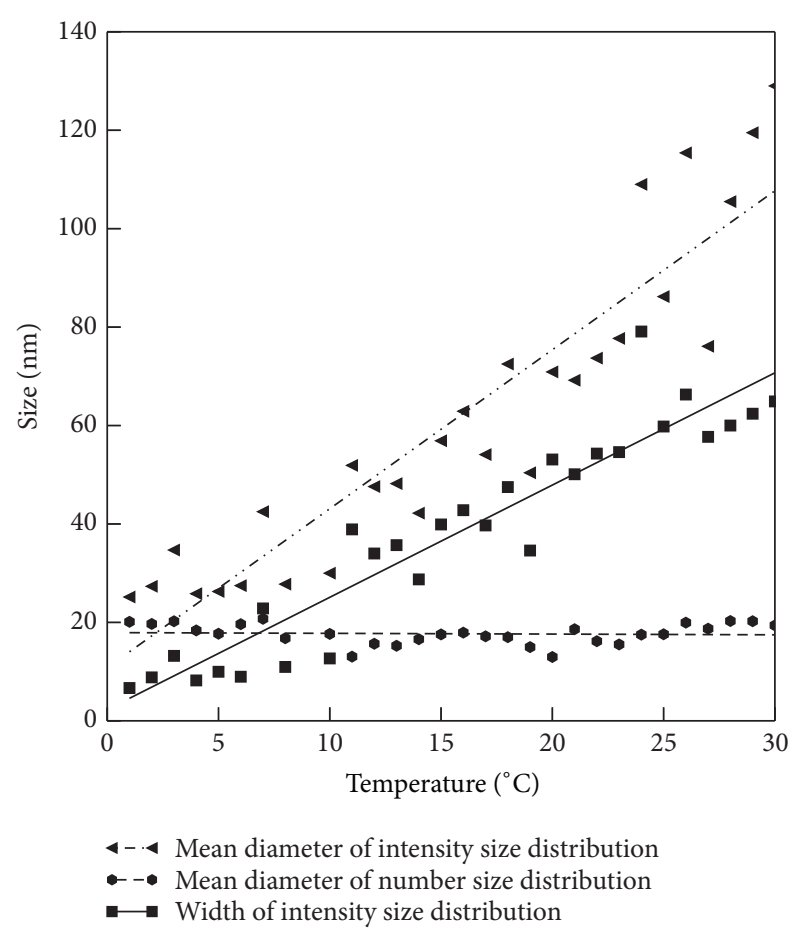

FIGURE 8: Dependence of the particle size distribution parameters on the synthesis temperature determined at the weight ratio $\mathrm{NaBH}_{4}: \mathrm{LYZ}: \mathrm{AgNO}_{3}=0.22: 0.77: 1$.

materials without additional purification and extraction of the necessary disperse particle fraction.

A problem of interest that calls for a subsequent, more profound study is the state and structure of a lysozyme molecule in the shell, and further research can be applied in the field of biosensors and drug development of a new generation.

\section{Conflict of Interests}

The authors declare that there is no conflict of interests regarding the publication of this paper.

\section{Acknowledgments}

The authors gratefully acknowledge Andriyanova A. Yu for great help during the experimental work. Also thanks are due to Maslennikova T. P. for assistance in the microscopic studies. Financial support was received from the Russian Foundation for Basic Research (Project no. 14-03-00626).

\section{References}

[1] M. Rai, A. Yadav, and A. Gade, "Silver nanoparticles as a new generation of antimicrobials," Biotechnology Advances, vol. 27, no. 1, pp. 76-83, 2009.

[2] N. Vigneshwaran, R. P. Nachane, R. H. Balasubramanya, and P. V. Varadarajan, "A novel one-pot "green" synthesis of stable silver nanoparticles using soluble starch," Carbohydrate Research, vol. 341, no. 12, pp. 2012-2018, 2006.
[3] A. L. González and C. Noguez, "Optical properties of silver nanoparticles," Physica Status Solidi C, vol. 4, no. 11, pp. 41184126, 2007.

[4] D. D. Evanoff Jr. and G. Chumanov, "Synthesis and optical properties of silver nanoparticles and arrays," ChemPhysChem, vol. 6, no. 7, pp. 1221-1231, 2005.

[5] L. Balan, J.-P. Malval, R. Schneider, and D. Burget, "Silver nanoparticles: new synthesis, characterization and photophysical properties," Materials Chemistry and Physics, vol. 104, no. 2-3, pp. 417-421, 2007.

[6] S. Shrivastava, T. Bera, S. K. Singh, G. Singh, P. Ramachandrarao, and D. Dash, "Characterization of antiplatelet properties of silver nanoparticles," ACS Nano, vol. 3, no. 6, pp. 1357-1364, 2009.

[7] M. Liang, L. Wang, R. Su et al., "Synthesis of silver nanoparticles within cross-linked lysozyme crystals as recyclable catalysts for 4-nitrophenol reduction," Catalysis Science and Technology, vol. 3, no. 8, pp. 1910-1914, 2013.

[8] O. Golubeva, O. Shamova, D. Orlov, E. Yamshchikova, A. Boldina, and V. Kokryakov, "Synthesis and investigation of silver-peptide bioconjugates and investigation in their antimicrobial activity," in Materials Challenges and Testing for Supply of Energy and Resources, T. Böllinghaus, J. Lexow, T. Kishi, and M. Kitagawa, Eds., pp. 163-171, Springer, Berlin, Germany, 2012.

[9] A. R. L. Caires, L. R. Costa, and J. Fernandes, "A close analysis of metal-enhanced fluorescence of tryptophan induced by silver nanoparticles: wavelength emission dependence," Central European Journal of Chemistry, vol. 11, no. 1, pp. 111-115, 2013.

[10] I. Sondi and B. Salopek-Sondi, "Silver nanoparticles as antimicrobial agent: a case study on E. coli as a model for Gramnegative bacteria," Journal of Colloid and Interface Science, vol. 275, no. 1, pp. 177-182, 2004.

[11] S. Chernousova and M. Epple, "Silver as antibacterial agent: ion, nanoparticle, and metal," Angewandte Chemie, vol. 52, no. 6, pp. 1636-1653, 2013.

[12] S. R. Mudshinge, A. B. Deore, S. Patil, and C. M. Bhalgat, "Nanoparticles: emerging carriers for drug delivery," Saudi Pharmaceutical Journal, vol. 19, no. 3, pp. 129-141, 2011.

[13] D. Joshi and R. K. Soni, "Laser-induced synthesis of silver nanoparticles and their conjugation with protein," Applied Physics A, vol. 116, no. 2, pp. 635-641, 2014.

[14] M. L. Gulrajani, D. Gupta, S. Periyasamy, and S. G. Muthu, "Preparation and application of silver nanoparticles on silk for imparting antimicrobial properties," Journal of Applied Polymer Science, vol. 108, no. 1, pp. 614-623, 2008.

[15] K. C. Song, S. M. Lee, T. S. Park, and B. S. Lee, "Preparation of colloidal silver nanoparticles by chemical reduction method," Korean Journal of Chemical Engineering, vol. 26, no. 1, pp. 153155, 2009.

[16] D. M. Eby, N. M. Schaeublin, K. E. Farrington, S. M. Hussain, and G. R. Johnson, "Lysozyme catalyzes the formation of antimicrobial silver nanoparticles," ACS Nano, vol. 3, no. 4, pp. 984-994, 2009.

[17] T.-H. Kim, M. Kim, H.-S. Park, U. S. Shin, M.-S. Gong, and H.W. Kim, "Size-dependent cellular toxicity of silver nanoparticles," Journal of Biomedical Materials Research Part A, vol. 100, no. 4, pp. 1033-1043, 2012.

[18] C. Carlson, S. M. Hussein, A. M. Schrand et al., "Unique cellular interaction of silver nanoparticles: size-dependent generation of reactive oxygen species," Journal of Physical Chemistry B, vol. 112, no. 43, pp. 13608-13619, 2008. 
[19] S. Prabhu and E. Poulose, "Silver nanoparticles: mechanism of antimicrobial action, synthesis, medical applications, and toxicity effects," International Nano Letters, vol. 2, pp. 1-10, 2010.

[20] S. F. Sweeney, G. H. Woehrle, and J. E. Hutchison, "Rapid purification and size separation of gold nanoparticles via diafiltration," Journal of the American Chemical Society, vol. 128, no. 10, pp. 3190-3197, 2006.

[21] J. Benavides, O. Aguilar, B. H. Lapizco-Encinas, and M. RitoPalomares, "Extraction and purification of bioproducts and nanoparticles using aqueous two-phase systems strategies," Chemical Engineering and Technology, vol. 31, no. 6, pp. 838845, 2008.

[22] V. S. Gurin, V. P. Petranovskii, and N. E. Bogdanchikova, "Metal clusters and nanoparticles assembled in zeolites: an example of stable materials with controllable particle size," Materials Science and Engineering C, vol. 19, no. 1-2, pp. 327-331, 2002.

[23] A. Taleb, C. Petit, and M. P. Pileni, "Synthesis of highly monodisperce silver nanoparticles from aot reverse micelles: a way to $2 \mathrm{~d}$ and $3 \mathrm{~d}$ self organization," Chemistry of Materials, vol. 9, no. 4, pp. 950-959, 1997.

[24] P. Mukherjee, A. Ahmad, D. Mandal et al., "Fungus-mediated synthesis of silver nanoparticles and their immobilization in the mycelial matrix: a novel biological approach to nanoparticle synthesis," Nano Letters, vol. 1, no. 10, pp. 515-519, 2001.

[25] R. Das, R. Jagannathan, C. Sharan, U. Kumar, and P. Poddar, "Mechanistic study of surface functionalization of enzyme lysozyme synthesized $\mathrm{Ag}$ and $\mathrm{Au}$ nanoparticles using surface enhanced Raman spectroscopy," Journal of Physical Chemistry C, vol. 113, no. 52, pp. 21493-21500, 2009.

[26] G. K. Chandra, D. R. Tripathy, S. Dasgupta, and A. Roy, "Interaction of (-)-epigallocatechin gallate with lysozyme-conjugated silver nanoparticles," Applied Spectroscopy, vol. 66, no. 7, pp. 744-749, 2012.

[27] T. Yang, Z. Li, L. Wang, C. Guo, and Y. Sun, "Synthesis, characterization, and self-assembly of protein lysozyme monolayerstabilized gold nanoparticles," Langmuir, vol. 23, no. 21, pp. 10533-10538, 2007.

[28] A. Panuszko, M. Wojciechowski, P. Bruździak, P. W. Rakowska, and J. Stangret, "Characteristics of hydration water around hen egg lysozyme as the protein model in aqueous solution. FTIR spectroscopy and molecular dynamics simulation," Physical Chemistry Chemical Physics, vol. 14, no. 45, pp. 15765-15773, 2012.

[29] E. Jones, T. Oliphant, P. Peterson et al., "SciPy: Open source scientific tools for Python," 2001, http://www.scipy.org.

[30] V. Amendola, O. M. Bakr, and F. Stellacci, "A study of the surface plasmon resonance of silver nanoparticles by the discrete dipole approximation method: effect of shape, size, structure, and assembly," Plasmonics, vol. 5, no. 1, pp. 85-97, 2010.

[31] G. Chandra, K. S. Ghosh, S. Dasgupta, and A. Roy, "Evidence of conformational changes in adsorbed lysozyme molecule on silver colloids,' International Journal of Biological Macromolecules, vol. 47, no. 3, pp. 361-365, 2010.

[32] J. Hu, R. S. Sheng, Z. S. Xu, and Y. Zeng, "Surface enhanced raman spectroscopy of lysozyme," Spectrochimica Acta A, vol. 51, no. 6, pp. 1087-1096, 1995.

[33] E. Podstawka, Y. Ozaki, and L. M. Proniewicz, "Adsorption of S-S containing proteins on a colloidal silver surface studied by surface-enhanced Raman spectroscopy," Applied Spectroscopy, vol. 58, no. 10, pp. 1147-1156, 2004.
[34] G. D. Chumanov, R. G. Efremov, and I. R. Nabiev, "Surfaceenhanced Raman spectroscopy of biomolecules. Part I.-watersoluble proteins, dipeptides and amino acids," Journal of Raman Spectroscopy, vol. 21, no. 1, pp. 43-48, 1990.

[35] B. Fischer, I. Sumner, and P. Goodenough, "Renaturation of lysozyme-temperature dependent of renaturation rate, renaturation yield, and aggregation: identification of hydrophobic folding intermediates," Archives of Biochemistry and Biophysics, vol. 306, no. 1, pp. 183-187, 1993. 

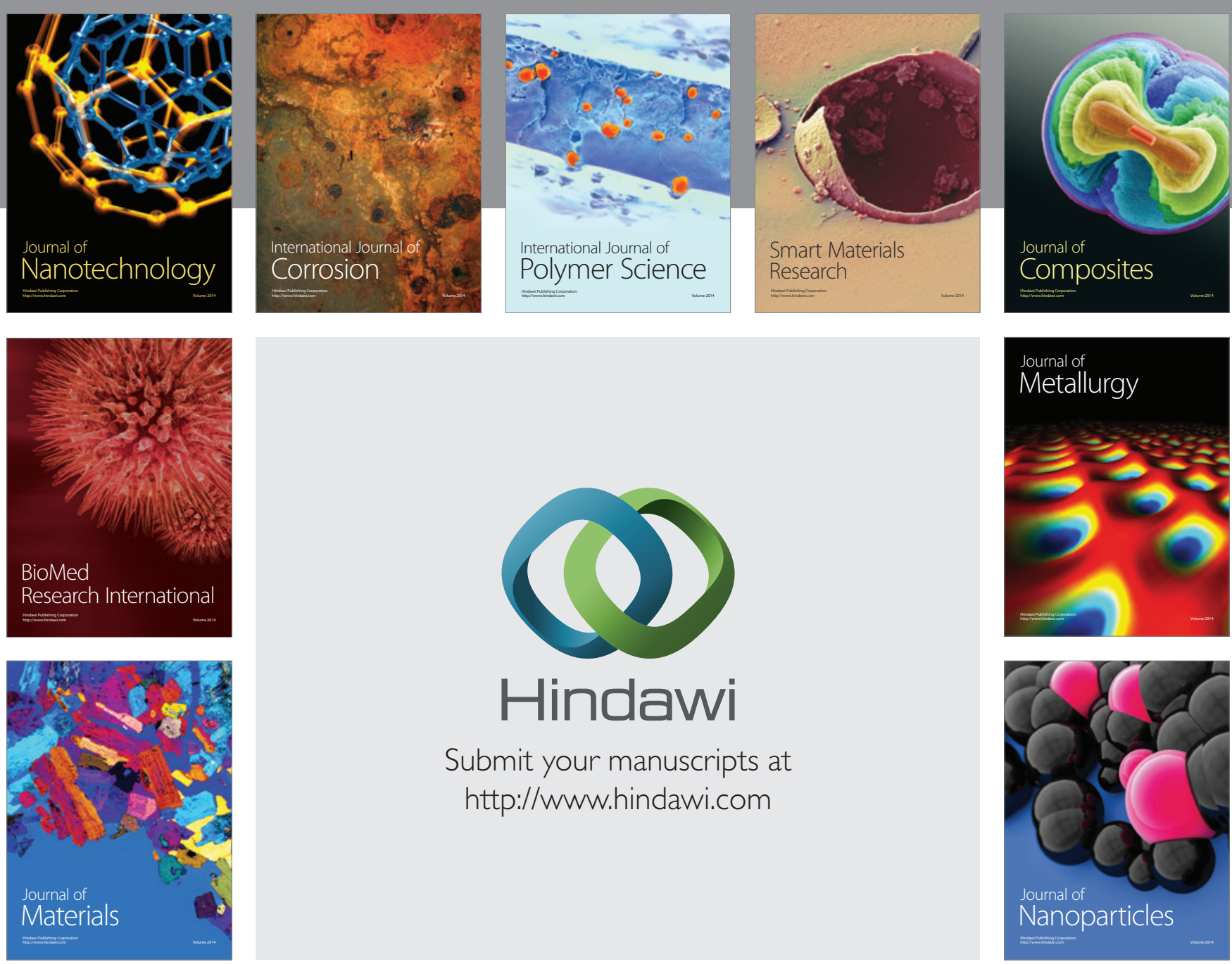

Submit your manuscripts at http://www.hindawi.com
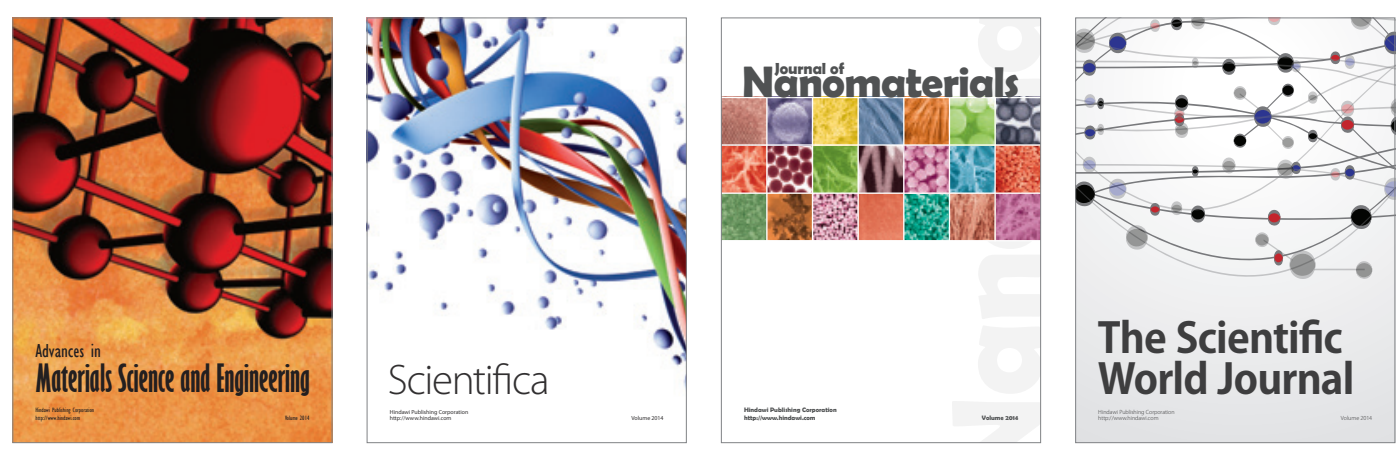

\section{The Scientific World Journal}
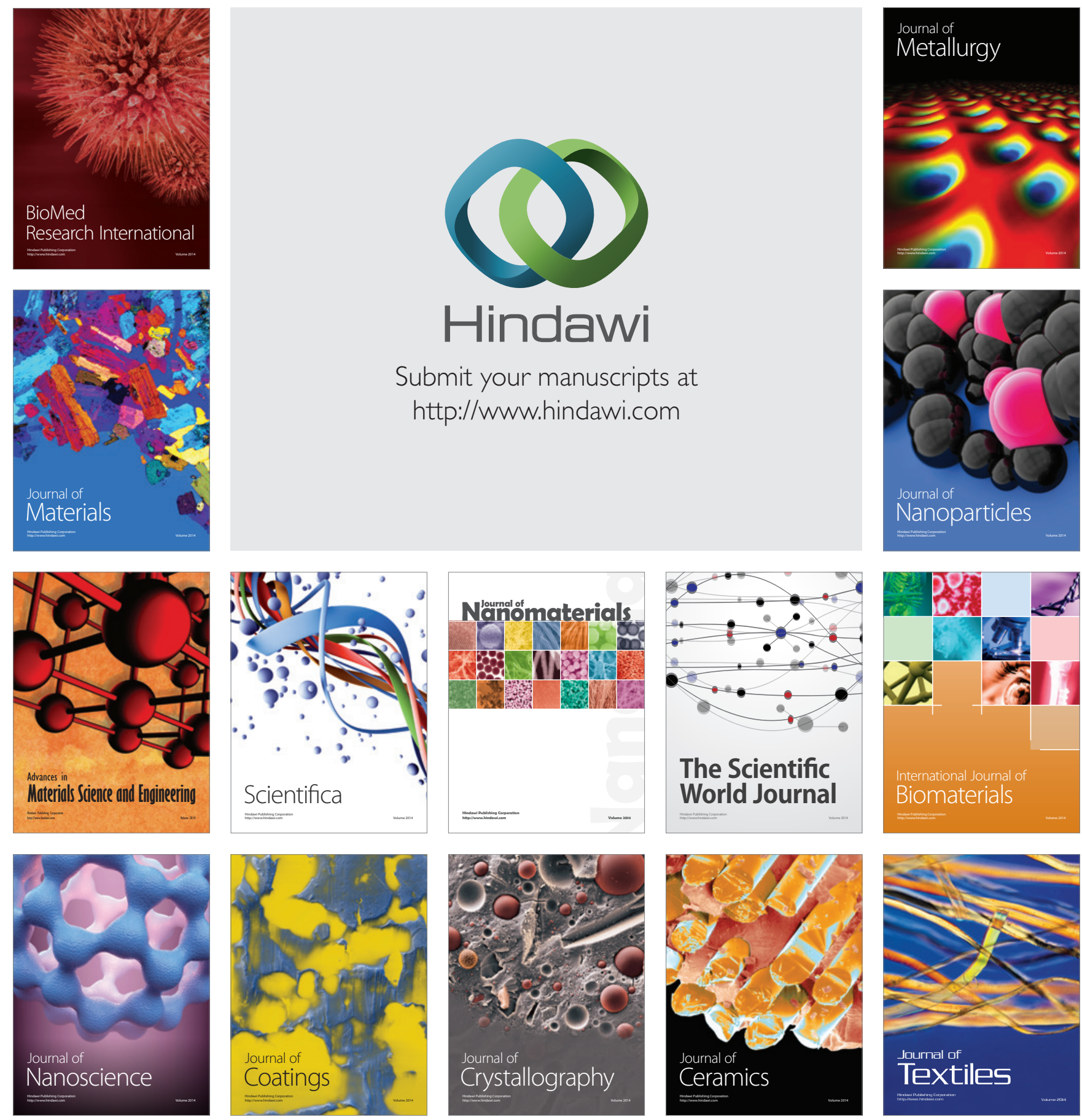